\title{
Salud Global en las instituciones académicas latinoamericanas: hacia un desarrollo $e$ identidad propia
}

\author{
Global Health in Latin-American academic institutions: \\ towards a distinctive development and identity
}

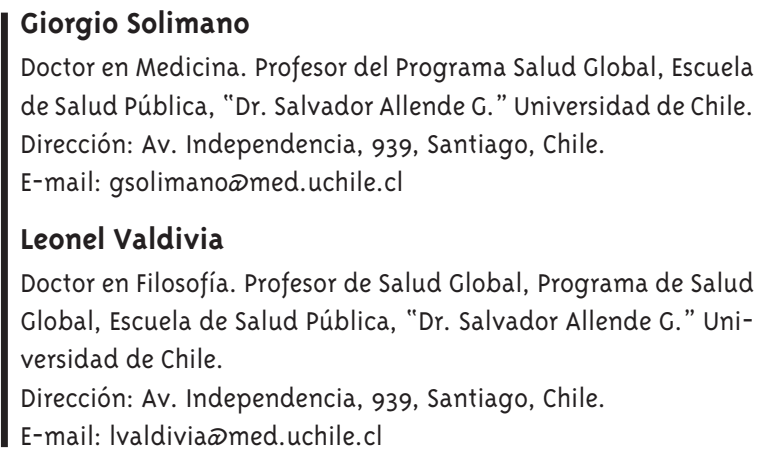

\section{Resumen}

Salud Global es un campo relativamente nuevo en las instituciones académicas de América Latina y su expansión ha sido lenta en comparación con el explosivo desarrollo que esta disciplina ha experimentado en países de Europa y Norteamérica. Este artículo presenta una visión general de los principales programas de docencia e investigación en Salud Global en universidades e institutos de la Región que han surgido durante los últimos 10 años. Asimismo, se describe la creación de ALASAG, una red de instituciones académicas con programas en Salud Global, a través de la cual se está haciendo sentir la voz de Latinoamérica en los foros mundiales de Salud Global. Los autores plantean que el quehacer de las instituciones académicas debe, necesariamente, inscribirse dentro de una conceptualización e identidad regional propia y distintiva. La perspectiva Latinoamericana de la Salud Global debe partir de una posición crítica frente al fenómeno de globalización económica capitalista manejado desde la ideología neoliberal. Desde dicha perspectiva se propone un listado de aéreas prioritarias en docencia, investigación y abogacía en Salud Global para la Región, entre otras: la equidad en el acceso a la salud; la globalización económica en América Latina y sus efectos en la equidad en salud; y la liberación y protección del comercio internacional en contraposición con la protección de la salud humana y el medio ambiente.

Palabras clave: Salud Global; América Latina; Globalización; Neoliberalismo; Equidad en Salud. 


\section{Abstract}

Global Health is a relatively recent development in Latin American academic institutions. Its expansion among such institutions has been slow in comparison with its explosive development in European and North American countries. This article presents an overview of the main Global Health teaching and research programs that have emerged over the past decade at universities and institutes of the Region. Likewise, it describes the creation of ALASAG, a network of academic institutions interested in Global Health, through which the voice of Latin America is being heard in world Global Health fora. The authors propose that the Global Health work at Latin American academic institutions should be based on a distinctive, Region-based, conceptual vision and identity of Global Health. Such Latin American vision must necessarily involve a critical stance on the capitalist economic globalization phenomenon inspired by the neoliberal ideology. From this critical stance, the authors propose a number of priority areas for Global Health teaching, research and advocacy for Latin America academic institutions including, among others, equitable access to health, economic globalization and its effect on health equity, and international trade liberalization and protection and its impact on the environment and human health. Keywords: Global Health; Latin America; Globalization; Neoliberalism; Health Equity.

\section{Desarrollo de la Salud Global en América Latina}

La Salud Global es aun un campo en construcción en América Latina. Durante la última década, está recibiendo creciente atención a nivel académico, de gobiernos, organismos internacionales y también de las organizaciones de la sociedad civil. Sin embargo, su perspectiva en nuestra Región, aun cuando se ha nutrido de conceptos generados en los países del hemisferio norte ha ido adquiriendo identidad propia, íntimamente relacionada con las características del desarrollo en nuestros países, en los cuales la desigualdad e inequidad afectan a sectores importantes de la población, incluso mayoritarios en algunos de ellos.

La formación y capacitación de profesionales de la salud y de otras disciplinas y el fortalecimiento de la investigación en un grupo de Escuelas e Institutos de Salud Pública, ha ganado espacio y legitimidad generando Programas o Centros de Salud Global.

En la actualidad, al menos tres instituciones tienen programas de Salud Global como parte de sus unidades académicas, el INSP en México, la ESP/UCH en Chile y la FSP/UPCH en Perú; a su vez la Fundación Fiocruz en Brasil cuenta con el Centro de Relaciones Internacionales en Salud (CRIS) que concentra su quehacer en las relaciones internacionales, especialmente desde la perspectiva de cooperación Sur-Sur y globalización. Por su parte las facultades y escuelas de las universidades de Antioquia en Colombia, de Costa Rica y de San Pablo en Brasil han establecido en los últimos años cursos, diplomas y maestrías instalando esta temática en sus mallas curriculares. Se espera que en el futuro próximo otras universidades sigan este camino.

Este escenario llevó el año 2010 a la constitución de la Alianza Latinoamericana de Salud Global (ALASAG) conformada actualmente por 8 prestigiosas Universidades latinoamericanas.

Igualmente, durante este período varios gobiernos han reconocido la importancia de Salud Global en diferentes ámbitos de su quehacer, tales como las relaciones internacionales y la diplomacia, el comercio exterior, la regulación de la industria de medicamentos y de alimentos, la acreditación de profesionales migrantes y la trasmisión inter países 
de enfermedades trasmisibles, por nombrar algunos temas prioritarios en este campo. Sin embargo, trabajar con organizaciones no gubernamentales y comunitarias, en el ámbito de la abogacía y la acción social en problemas relacionados con Salud Global, hasta ahora solo ha sido abordado de manera marginal por nuestras instituciones.

A continuación nos referiremos a las principales iniciativas académicas implementadas por las Facultades, Escuelas e Institutos que forman parte de ALASAG, así como a los objetivos y actividades de la ALASAG propiamente tal.

La educación en Salud Global en Latinoamérica hizo su aparición a comienzos de la década pasada y se estima que en la actualidad no más de 10 universidades imparten docencia en este campo. En una reunión convocada por OPS/OMS el año 2009 para discutir un nuevo modelo conceptual sobre salud internacional, hubo consenso que la Salud Global tenía un gran potencial de crecimiento y de colaboración en América Latina, ya que ella permite comprender mejor los factores globales que influyen en la salud de la población en un contexto de profundos cambios sociales y económicos. El año 2003 el Instituto Nacional de Salud Pública de México realizó el primer curso sobre Salud Global con apoyo de la Fundación Fullbright, titulado "Desafíos de la Globalización y Salud en América Latina”, en el cual participaron docentes de cinco continentes. Desde entonces el INSP ha continuado ofreciendo el curso "Fundamentos de la Salud Global en Latinoamérica". El año 2006, luego de obtener una donación del Centro Internacional Fogarty, el INSP creó el Programa de Salud Global, lo que le ha permitido dictar desde el año 2009 un Diploma en Salud Global, que atrae estudiantes de México y de otros países de la Región. También ha desarrollado una línea de formación en Salud Global para estudiantes regulares de los programas de maestría y doctorado y ha asignado becas para estadías cortas en el extranjero (Salgado, 2010).

La Escuela de Salud Pública de la Universidad de Chile creó el Programa de Salud Global en el año 2010, ofreciendo, desde entonces, en la Escuela Internacional de Verano siete cursos sobre diferentes temas de Salud Global y un Diploma para Graduados, en los cuales han participado académicos de Canadá, Estados Unidos y Nueva Zelanda, entre otros. Este año se ofrecerá un Seminario orientado a analizar los problemas clave en Salud Global en América latina, con el propósito de construir una agenda que apoye las políticas públicas en este campo. En 2014 se dictará un curso interdisciplinario para estudiantes de Doctorado, Magister y Médicos Especialistas en Salud Pública de la Universidad de Chile y de otras universidades que expresen interés (Solimano y Valdivia, 2011).

La Facultad de Salud Pública y Administración de la Universidad Peruana Cayetano Heredia, ofrece desde el año 2005 el curso "Conceptos Básicos en Salud Global”, con el propósito de entregarle a estudiantes de pre y postgrado una perspectiva de los problemas que enfrenta Perú en este campo. En conjunto con la Universidad de Washington el año 2012 realizaron el curso "Salud Internacional, Salud Global y Diplomacia" para profesionales de salud del Pacto Andino y del Ministerio de Salud de Perú, responsables de la cooperación internacional. Además, esta facultad dicta una Maestría en Salud Pública y Salud Global de veinte meses de duración.

En Brasil, tanto la Escuela Nacional de Salud Pública/Fiocruz de Río de Janeiro como la Facultad de Salud Pública de la Universidad de San Pablo, realizan actividades en Salud Global. La primera dictó en el año 2009 un curso sobre "Salud Global y Diplomacia”, enfatizando el impacto de la globalización sobre la salud y de la cooperación internacional en las políticas de salud de Brasil. Recientemente la ENSP/Fiocruz ha implementado un Diploma y una Maestría Profesional en Salud Global. Por su parte, la FSP/USP, realizó en 2012 un primer curso en Salud Global sobre "Bioética y Salud Global” y a partir de 2013 ofrecerá un Doctorado en Salud Global y Sustentabilidad.

El examen de la educación en Salud Global que se imparte en las universidades latinoamericanas, permite concluir que ella tiene características que la diferencian de la que se entrega en Norteamérica y algunos países de Europa. En primer lugar, los cursos se focalizan preferentemente en los efectos de la globalización en la salud poblacional y las políticas y sistemas de salud de los países que los dictan, así como en la región latinoamericana. Igualmente, se privilegia el pensamiento y análisis crítico de la institucionalidad y las estructuras de 
poder dominantes en la sociedad contemporánea. Otra característica de nuestros programas de enseñanza es la utilización de tecnologías de la información y la comunicación (TICS), que permite realizar educación a distancia y por lo tanto llegar a una audiencia más amplia. La mayoría de los cursos están dirigidos a estudiantes graduados en salud pública y profesionales interesados en educación continua, siendo la docencia en pregrado una de las tareas pendientes. La colaboración interinstitucional e internacional es otra característica destacable de la enseñanza en Salud Global en nuestra región (Missoni y Tediosi, 2013).

El desarrollo de la investigación en Salud Global ha sido más lento, careciendo de una identidad clara en término de líneas de investigación y de una masa crítica de investigadores. En este contexto, el INSP de México es la institución que cuenta con mayor desarrollo en este campo, identificando los proyectos que realizan los diferentes Centros de Investigación del Instituto “con enfoque en Salud Global”. Por su parte la FSP/USP ha definido dos líneas de investigación en Salud Global, y la UPCH/Perú ha contado con el apoyo del Fogarty International Center para realizar sus proyectos de investigación. En la Universidad de Chile varias propuestas sometidas a financiamiento internacional, tales como CYTED, Unión Europea, CIRH, no han sido aprobadas o están en proceso de revisión.

La constitución de alianzas, asociaciones y redes han permitido establecer relaciones de colaboración impensadas hace 20 años en América Latina. Ellas son parte de la globalización y bien utilizadas pueden contribuir significativamente al desarrollo social y económico de nuestras sociedades. La salud no es una excepción en este escenario.

La Alianza Latinoamericana y del Caribe de Salud Global (ALASAG), creada en el año 2010, constituye una red de instituciones académicas de colaboración Sur-Sur, surgiendo como una respuesta para enfrentar riesgos comunes de salud de sus poblaciones. La Alianza se basa en las realidades nacionales y en un profundo respeto a la idiosincrasia e identidad de sus pueblos. Igualmente, aprovecha las nuevas concepciones de cooperación internacional que dinamiza las colaboraciones sur - norte y norte - sur en el campo de la Salud Global.
El Acta de Constitución de ALASAG, firmada en Santiago de Chile con ocasión del $2^{\circ}$ Congreso Latinoamericano y del Caribe sobre Salud Global, establece como misión "Impulsar el abordaje de Salud Global en la enseñanza, capacitación, investigación, vinculación y cooperación técnica en Latinoamérica y el Caribe a través de colaboraciones interinstitucionales" (ALASAG, 2013a, p. 1) y como visión "Ser la alianza líder en Salud Global en América Latina y el Caribe y portavoz del tema en la región y a nivel mundial" (ALASAG, 2013a, p. 1). Sobre la base de una asociación voluntaria de instituciones académicas, ALASAG se propone hacer realidad los siguientes objetivos:

- "Promover la cooperación internacional utilizando modelos que integren diversas disciplinas, sectores, niveles y métodos que generen sinergias y colaboraciones en temas de Salud Global prioritarios para América Latina y el Caribe”.

- "Aprovechar las nuevas dinámicas de cooperación que enfatizan un ambiente de colaboración regional, especialmente entre las naciones del sur, y potenciando colaboraciones con flujos de sur a norte y de norte a sur en las áreas de Salud Global en la investigación, formación y capacitación de recursos humanos, vinculación y cooperación técnica”.

- "Contribuir a la institucionalización del Congreso Latinoamericano y del Caribe sobre Salud Global como el foro oficial de ALASAG, con una periodicidad bianual" (ALASAG, 2013a, p. 1).

Durante su corta existencia ALASAG ha realizado dos congresos, el primero el año 2010 en el INSP/ México y el segundo en Santiago de Chile el año 2013. Este último convocado por la Alianza Latinoamericana para la Salud Global y la Escuela de Salud Pública, Facultad de Medicina de la Universidad de Chile tuvo como lema del Congreso "Trascendiendo Fronteras para la Equidad en Salud”. En él participaron alrededor de 700 investigadores y profesionales de la Salud Global de América Latina, Norte América y Europa en conferencias magistrales, paneles y simposios simultáneos y la presentación de 300 trabajos científicos y experiencias innovadoras (ALASAG, 2013b).

Como culminación del Congreso se aprobó la Declaración de Santiago la que expresa: 
ALASAG entiende la Salud Global como un bien público mundial que trasciende fronteras, que está relacionado con la justicia social y que tiene como ejes a la equidad, la ética y el respeto a los derechos humanos. Buscamos la cooperación entre las instituciones miembros en la formación de recursos humanos, capacitación, investigación para la acción, cooperación técnica y abogacía en Salud Global, aprovechando similitudes, sinergias, necesidades e intereses compartidos en América Latina. Proponemos aportar la mirada latinoamericana a los temas más candentes de la agenda mundial sobre salud y desarrollo (ALASAG, 2013b).

Igualmente

Convocamos a los gobiernos, a los organismos multilaterales, a las instituciones académicas, a las organizaciones no gubernamentales, y otras organizaciones de la sociedad civil, a implementar estrategias que defiendan los intereses de las personas en términos de bienestar y desarrollo humano con el fin de lograr los postulados de la Salud Global. Estamos convencidos que el abordaje de la Salud Global debe sentar sus bases en el desarrollo de políticas públicas actuando sobre las necesidades reales de nuestra población y sobre los múltiples determinantes sociales de la salud que enfrentamos en la región (ALASAG, 2013b).

Luego de aprobarse el Plan de Acción para el período 2013-2014 los miembros de ALASAG están elaborando su reglamento y trabajando en el diseño e implementación de un diploma internacional sobre Salud Global con el propósito de formar, en plazo de tres años, una masa crítica de recursos humanos, adecuadamente capacitados en el campo de Salud Global, en las instituciones académicas de América Latina.

Simultáneamente con el fortalecimiento a nivel regional se han establecido relaciones con otros referentes internacionales tales como el Consorcio de Universidades para la Salud Global (CUGH), que tuvo una activa participación en ambos congresos latinoamericanos. A través de su Secretaría Técnica, ALASAG está participando activamente en la creación de la Federación Mundial de Instituciones Académicas para la Salud Global que tuvo su lanzamiento oficial en la Cumbre Mundial de la Salud realizada en Berlín en octubre 2013.
Así el balance de estos 10 años de trabajo de las instituciones académicas en América Latina puede considerarse positivo y se espera que nuevas instituciones se incorporen a este campo. Además nuestra Región, a través de ALASAG, está haciendo sentir su voz en los foros mundiales de Salud Global.

\section{Conceptualización y perspectivas de la Salud Global para América Latina}

El quehacer de las instituciones académicas anteriormente descrito se inscribe dentro de una necesaria conceptualización y perspectiva latinoamericana que la diferencia, y le da una identidad propia. Es el tema que abordaremos en esta sección.

No cabe duda que el campo de la Salud Global (al igual que sus antecesores Salud Internacional y Salud Tropical) se originó y aun recibe su impulso principalmente desde el Norte. Este es el Norte Global, que incluye algunos países localizados en el Hemisferio Sur, mientras en el Sur Global, constituido por países de menor desarrollo económico y social, estos han sido principalmente receptores de colaboración y anfitriones de consultores, investigadores y estudiantes en busca de experiencias primarias respecto a los temas de este campo. Al revisar la literatura internacional sobre salud en general y Salud Global, en particular, es evidente el avance explosivo de esta última, constituyendo las publicaciones en idioma inglés, la mayoría. Asimismo destaca la presencia de la Salud Global como un campo prioritario de docencia e investigación en las universidades norteamericanas y europeas. Prácticamente todas las escuelas de salud pública de las principales universidades estadounidenses cuentan con programas de Salud Global, teniendo, al parecer, como propósito evidente el atraer un mayor número de estudiantes tanto del país como del extranjero (MacFarlane y col., 2008).

Tanto en las instituciones académicas como en las diversas organizaciones internacionales, gubernamentales y no gubernamentales, el discurso sobre Salud Global varía del tradicional altruismo de aliviar los problemas de salud de la población en los países pobres (similar al discurso de la Salud Internacional de principios y mediados del siglo XX), a 
un discurso más auto referente de proyectarse como instituciones de excelencia en investigación y docencia en salud en el mundo. En parte, el surgimiento y rápido desarrollo del campo de la Salud Global, se debe a la demanda de las nuevas generaciones que desean conocer el mundo y sus problemas más allá de las fronteras de sus países. Un ejemplo visible del creciente interés en Norteamérica es el rápido desarrollo del Consorcio de Universidades para la Salud Global (CUGH) (y su predecesor el Consorcio de Educación para la Salud Global - GHEC), una red de instituciones académicas que en cinco años ha aumentado su membrecía a más cien universidades de USA y Canadá y la concurrencia a sus reuniones anuales llega a varios miles de participantes. Similares redes de instituciones académicas en Salud Global se han establecido en países y regiones europeas.

En América Latina la situación es bastante diferente. Aunque desde hace varios años, universidades e institutos de nuestra región colaboran con instituciones del Norte en la realización de investigación y docencia sobre temas de Salud Global, la búsqueda de un desarrollo propio en este campo con una perspectiva e identidad regional es relativamente nueva (Franco, 2003; Franco y Álvarez-Dardet, 2009).

Formalmente, como se mencionó en la sección anterior, fue durante el Primer Congreso Latinoamericano y del Caribe sobre Salud Global realizado en México en abril de 2010, que un grupo de representantes de instituciones académicas latinoamericanas decidió constituirse en una red bajo el nombre de Alianza Latinoamérica para la Salud Global (ALASAG, 2013a), actuando el Instituto Nacional de Salud Publica de México como su Secretaría Técnica. Hasta el presente, ALASAG ha tenido un desarrollo totalmente endógeno en América Latina, no habiendo recibido aportes de fuera de la Región. A pesar de ello se logró realizar el Segundo Congreso Latinoamericano y del Caribe sobre Salud Global en Santiago de Chile en enero 2013.

La Salud Global, como materia de docencia e investigación forma parte de un proceso de cambio de época, marcado por la transición desde las tensiones de la polarización de la Guerra Fría a las de la Globalización, transición atribuida al triunfo del capitalismo y la economía de libre mercado, con el predominio ideológico del sistema neoliberal
(Schrecker y col., 2008). En la década de los 7o, Chile, gobernado por una dictadura militar represiva, se incorporó tempranamente al neoliberalismo. Las consecuencias de la adhesión chilena al sistema de libre mercado en el campo de salud no se dejaron esperar. Las reformas profundas desde un Servicio Nacional de Salud creado en la década de los 5o, dio paso a la privatización del financiamiento y la prestación de servicios de salud y de previsión social. Por tanto, consideramos indispensable que el abordaje de la Salud Global, en Chile y América Latina, debe tomar como punto de partida una caracterización del fenómeno de la globalización económica y su impacto en la equidad en el acceso a servicios de salud y el bienestar general de la población.

Hasta ahora, el campo de la Salud Global ha sido de carácter predominantemente Euro y Norte céntrico, es decir que tanto su estudio como sus acciones han estado dirigidas desde instituciones de los países hegemónicos del mundo capitalista; por lo tanto, no sorprende que el discurso tenga también características hegemónicas y auto referentes. Aunque el tema de las desigualdades en salud, al interior y entre países, se está haciendo más presente en el discurso, en lo fundamental este aún no incluye un análisis crítico de las causas estructurales de dichas desigualdades. Dicho análisis tiende a excluir el tema político sobre quienes detentan y ejercen del poder. En otras palabras, el discurso de la Salud Global es aún demasiado parecido al de la Salud Internacional tradicional.

En un país como Chile donde predomina el mercado en salud, en educación y en previsión social, los postulados sobre Salud Global deben ser claros en su crítica al lucro (salud como mercancía), en los efectos de la liberalización del comercio y en el impacto de los monopolios que controlan la industria farmacéutica, etc. En palabras de Schrecker y colaboradores (2008), se debe tomar en serio la naturaleza "inherentemente des-igualitaria de la globalización” (p. 1670) en sus conductas, sus reglas y sus valores (Labonté y col., 2007; Birdsall, 2006). Por sus vivencias históricas como satélites del sistema capitalista y por la tradición progresista de sus principales instituciones académicas, América Latina está llamada a enriquecer el debate sobre Salud Global a nivel planetario. Esto es lo 
que estamos buscando a través de redes institucionales como ALASAG. En otras palabras, América Latina está posicionada estratégicamente para dar un salto cualitativo en el pensar y el actuar sobre Salud Global, no solo a nivel regional sino también global. Nuestra región superó el colonialismo hace dos siglos y está consciente de que pueda emerger el neocolonialismo. Además, existe una larga tradición de cooperación entre países, la creación de instituciones como OSANPAN y luego OPS y de centros históricos de formación en Salud Pública en Brasil, México, Colombia y Chile entre otros datan desde mediados del Siglo XX. Por su parte la participación ciudadana que se expresa en movimientos sociales fuertes, visibles y cada vez más efectivos demanda un desarrollo más igualitario. Poco a poco los países de Región han logrado superar su dependencia de la ayuda externa, focalizando sus esfuerzos en la cooperación Sur-Sur y en relaciones equitativas de cooperación con instituciones del Norte.

Establecer una identidad regional propia, contextualizada en la realidad social, histórica, política y económica de los países de la región, con el fin de abordar de manera efectiva las desigualdades existentes al interior y entre los países, constituye uno de los desafíos prioritarios para Salud Global en Latinoamérica. Esta es una región que a pesar de su heterogeneidad comparte muchas similitudes, una de las cuales es que constituye la región más desigual del mundo debido a problemas estructurales, lo que genera severos efectos en las condiciones de salud de los individuos, las comunidades, los países y la región. En ese sentido, formular políticas que creen efectivas relaciones sur-sur y plantearse como iguales frente a los enfoques predominantemente asistencialistas de los países del norte, constituye otro desafío ineludible. Así mismo, hablar de Salud Global significa necesariamente discutir sobre equidad y justicia social, e incorporar la participación social, la inter sectorización y la cooperación internacional entre países.

Es dentro de este escenario, que proponemos las siguientes áreas prioritarias para fortalecer la investigación, la docencia y la abogacía en Salud Global con un enfoque Latinoamericano, que contribuya a la formulación de políticas públicas conducentes al logro de mayores niveles de equidad y justicia social:
- La equidad en el acceso a la salud, derivada de la justicia social, como imperativo ético de la Salud Global.

- La caracterización del fenómeno de la globalización económica en América Latina, y sus efectos en la equidad en acceso a la salud y el bienestar de los pueblos de la Región (Buss, 2007; Marmot y Wilkinson, 2001; WHO, 2008).

- La cooperación Sur-Sur en América Latina, logros y limitaciones: OEA, Pacto Andino, Grupo de Rio, MERCOSUR, UNASUR, etc.

- La salud como mercancía versus la salud como derecho. Implicancias para el logro de la cobertura universal.

- "Pacientes versus patentes", la protección de los derechos de propiedad intelectual y su impacto en el acceso a medicamentos (Fortín, 2008).

- El análisis crítico de la adscripción y cumplimiento por parte de nuestros gobiernos, a los convenios internacionales en pro de la salud y el bienestar de los pueblos, ej. Convención sobre Tabaco, UNITAID, Convenio OIT 169 sobre pueblos originarios, normativas internaciones de protección del medio ambiente, derechos laborales, protección de migrantes, derechos de la mujer, etc.

- La salud y el desarrollo sustentable, la protección del medio ambiente, la urbanización humanizada, los asentamientos humanos dignos, la políticas de vivienda.

- El rescate y valoración de la memoria histórica de la salud pública y la cooperación latinoamericana desde nuestros orígenes como naciones independientes.

- América Latina en el mundo: mejorando nuestro conocimiento y vínculos solidarios con el resto del mundo especialmente a través de colaboración Sur-Sur.

- El análisis crítico de los programas de ayuda externa (caridad versus derechos), búsqueda de nuevos enfoques en la colaboración Norte-Sur y Sur-Sur.

- Las crisis financieras y salud: políticas de austeridad y sus efectos en la salud.

Finalmente, el establecimiento de una agenda 
propia, que difiere de la de los países hegemónicos, constituye otra prioridad. Dicha agenda necesariamente debe incluir: el libre comercio y los tratados de comercio internacional; la legislación internacional sobre patentes de medicamentos; las normativas y regulaciones sobre la formación, retención y migración de profesionales de salud; el abordaje de los determinantes sociales de la salud con el fin de superar las inequidades existentes; y los riesgos epidemiológicos, tanto de enfermedades trasmisibles nuevas y emergentes como de crónicas no trasmisibles, son las más importantes.

A esta tarea deben sentirse convocadas y comprometidas todas las instituciones académicas de salud latinoamericanas ya que ellas, sin duda, pueden y deben contribuir al mejoramiento de las condiciones de salud y bienestar de nuestros pueblos.

\section{Referencias}

\begin{abstract}
ALASAG - ALIANZA LATINOAMERICANA DE SALUD GLOBAL. Acta de constitución. Santiago, 2013a. Disponible en: <http://www.cebes.org.br/ internaEditoria.asp? param $=$ click\&idArquivo $=448>$. Acceso en: 05 mayo 2014.
\end{abstract}

\section{ALASAG - ALIANZA LATINOAMERICANA DE SALUD GLOBAL. Declaración de Santiago.} Santiago, 2013b. Disponible en: <http://www. saludglobalinsp.mx/index.php?option=com content\&view=article $\& i d=269$ : dec-santiago $\&$ catid =39: noticiasrecientes\&Itemid=349>. Acceso en: 05 mayo 2014.

BIRDSALL, N. The world is not flat: inequality and injustice in our global economy. Helsinki: World Institute for Development Economics Research, 2006. (Wider Annual Lecture 2005). Disponible en: <http://www.wider.unu.edu/publications/annuallectures/en_GB/AL9/_files/78121127186268214/ default/annual-lecture-2005.pdf >. Acceso em: 15 agosto 2013 .

BUSS, P. M. Globalização, pobreza e saúde. Ciência \& Saúde Coletiva, Rio de Janeiro, v. 12, n. 6, p. 15751589, 2007.
FORTÍN, C. Régimen jurídico del comercio internacional y derechos humanos: una compleja relación. Anuario de Derechos Humanos, Santiago, n. 4, p. 231-244, 2008. Disponible en: <http://www. anuariocdh.uchile.cl/index.php/ADH/article/ download/13662/13944>. Acceso en: 05 mayo 2014. FRANCO, A. Globalizar la salud. Gaceta Sanitaria, Barcelona, v.17, n. 2, p. 157-163, 2003. Disponible en: <http://scielo.isciii.es/scielo.php? script=sci_ arttext\&pid=So213-91112003000200011\&lng=es\&n $\mathrm{rm}=$ iso $>$. Acceso en: 14 agosto 2013.

FRANCO, A.; ÁLVAREZ-DARDET, C. Salud pública global: un desafío a los límites de la salud internacional a propósito de la epidemia de influenza humana. Revista Panamericana de Salud Pública, Washington, DC, v. 25, n. 6, p. 540547, 2009.

LABONTÉ, R. et al. Towards health-equitable globalization: rights, regulation and redistribution: final report to the Commission on Social Determinants of Health. Ottawa: University of Ottawa, Institute of Population Health, 2007. Disponible en: <http://www.who. int/social_determinants/resources/gkn_final_ report_042008.pdf >. Acceso en: 12 agosto 2013.

MacFARLANE, S. et al. In the name of global health: trends in academic institutions. Journal of Public Health Policy, Basingstoke, v. 29, n. 4, p. 383-401, 2008.

MARMOT, M.; WILKINSON, R. G. Psychosocial and material pathways in the relation between income and health: a response to Lynch et al. BMJ, London, v. 322, p. 1233-1236, May 2001.

MISSONI, E.; TEDIOSI, F. (Ed.). Education in global health policy and management. Milano: Egea, 2013.

SALGADO, N. El programa de salud global del Instituto Nacional de Salud Pública. México: INSP, 2010.

SCHRECKER, T.; LABONTE, R; DE VOGLI, R. Globalization and health: the need for a global vision. The Lancet, London, v. 372, p. 1670-1676, Nov. 2008. 
SOLIMANO, G.; VALDIVIA, L. Programa de salud

global. Santiago: Universidad de Chile, Facultad de

Medicina, Escuela de Salud Pública, 2011.

WHO - WORLD HEALTH ORGANIZATION. Closing

the gap in a generation: health equity through

action on the social determinants of health.

Geneva, 2008. Disponible en: <http://whqlibdoc.

who.int/publications/2008/9789241563703_eng.

pdf $>$. Acceso en: 10 agosto 2013. 